\title{
BAROCEPTOR RESPONSES TO ACUTE RHYTHM CHANGES
}

\author{
BY \\ SHEILA HOWARTH* AND E. P. SHARPEY-SCHAFER \\ From the Department of Medicine, St. Thomas' Hospital \\ Received January 4, 1956
}

There is now evidence that the response of the peripheral resistance to acute changes in the stroke output of the heart is determined by baroceptor reflexes, since it has been shown in cats (Ead et al., 1952) and in man (Sharpey-Schafer, 1953) that these receptors respond to arterial pulse pressure as well as mean pressure changes. In normal man when the effective filling pressure of the heart is reduced by the Valsalva manœuvre or the erect posture, the subsequent decrease in stroke output and arterial pulse pressure is followed by peripheral vasoconstriction. It is more difficult to increase suddenly the normal stroke output and pulse pressure; but it has been shown that imposed cough transients are followed by peripheral vasodilatation (Sharpey-Schafer, 1953), which also results from the increased pulse pressure during squatting (Sharpey-Schafer, 1956).

It seemed possible that spontaneous acute changes in cardiac rhythm might provide additional evidence, since pulse pressure may alter conspicuously with sudden slowing or quickening of the heart beat.

\section{MATERIAL AND MethodS}

Two groups of cases were studied. In the first group, seven cases of paroxysmal tachycardia or flutter were chosen for short frequent bursts of ectopic rhythm occurring in an otherwise normal heart. A second commoner group of seventeen cases with heart disease and heart failure were also studied.

Intravascular pressures were recorded with capacitance manometers and forearm flow with the venous occlusion plethysmograph.

\section{Group I}

\section{RESULTS}

Effects of Sudden Slowing. Continuous arterial records during the change from a fast ectopic rhythm to sinus rhythm were obtained in five cases. The results were the same in all. The pulse pressure of the first normal beat was greatly increased when compared with the pulse pressure during the ectopic rhythm and the mean pressure of this beat was unaltered. Thereafter, however, diastolic and mean pressure decreased over three or four beats (Fig. 1). In two patients forearm flow was measured before and directly after the change to sinus rhythm. The flows after the change to sinus rhythm were 2.4 and 2.9 times respectively the flows before the rhythm change. The decrease in mean arterial pressure and increase in forearm flow indicate vasodilatation.

Effects of Sudden Rapid Beating. The onset of an ectopic rhythm was recorded four times, with similar results in all. There was an immediate and conspicuous decrease in pulse pressure. Mean pressure either remained constant or after a few beats began to fall slightly. After about fifteen beats of the rapid rhythm, however, mean pressure rose indicating the onset of vasoconstriction (Fig. 2).

\footnotetext{
* Formerly Senior Research Fellow, Institute of Cardiology.
} 


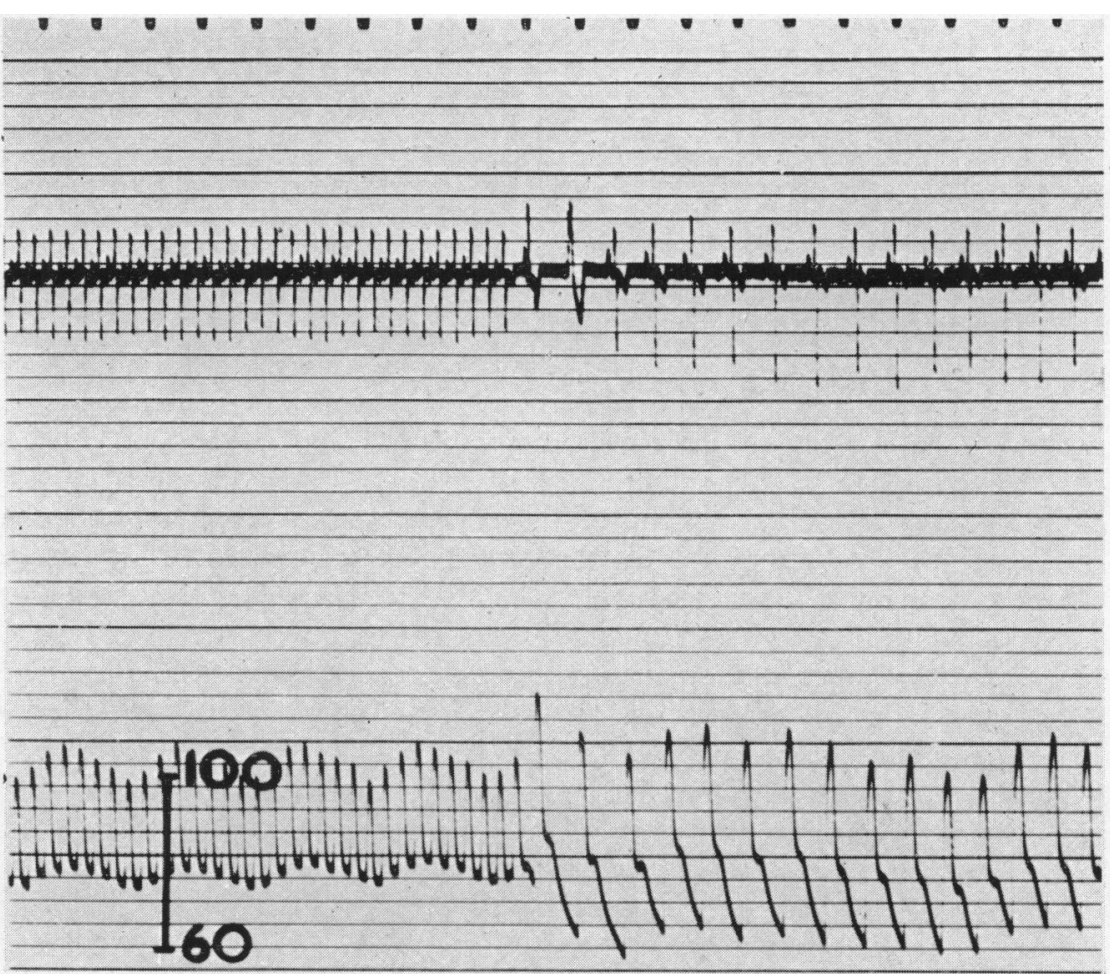

FIG. 1.-Upper trace electrocardiogram. Lower trace arterial pressure. Time marker in seconds and calibration in $\mathrm{mm}$. $\mathrm{Hg}$ in this and subsequent figures. The increased pulse pressure with the change to sinus rhythm caused vasodilatation and a fall in mean arterial pressure.

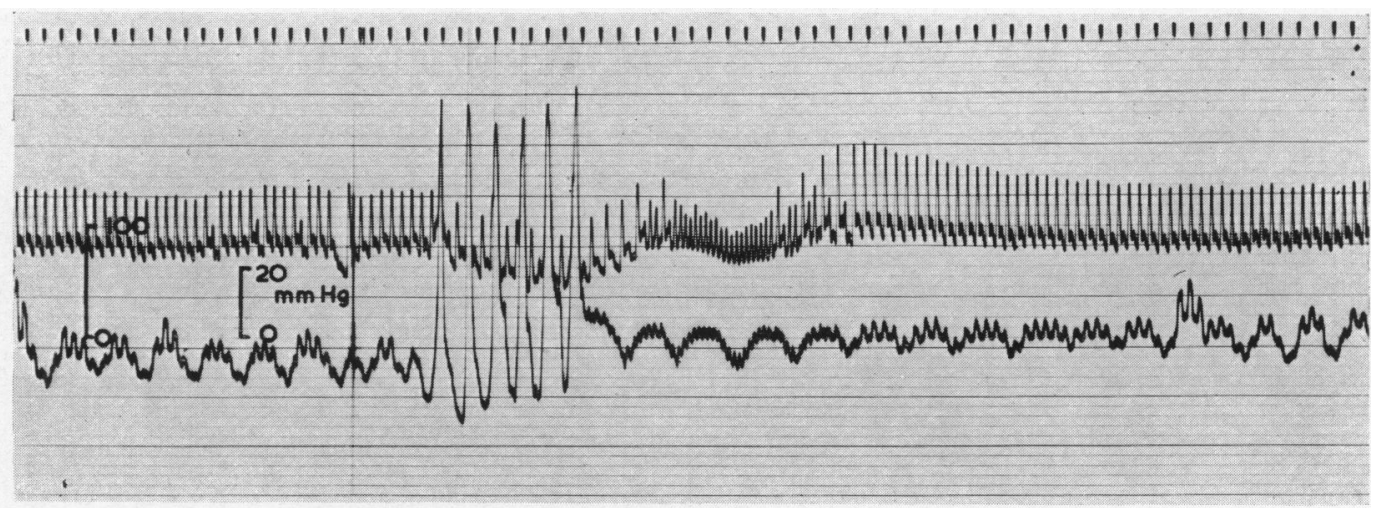

FIG. 2.-Upper trace arterial pressure. Lower trace intrathoracic (œesophageal) pressure. Three seconds after 6 cough transients there was a burst of fast ectopic rhythm. After a further 5 seconds the rise in mean arterial pressure indicates the onset of vasoconstriction. 
Acute Rhythm Change under a Sympathetic Blocking Agent. Fig. 3 shows a record from a patient who was known to have paroxysmal tachycardia, but whose circulation was otherwise normal. In the course of a surgical procedure $50 \mathrm{mg}$. of hexamethonium were given intravenously and the record obtained shortly after. It will be seen that in spite of a conspicuous decrease in pulse pressure with onset of the ectopic rhythm there is no evidence of vasoconstriction. The change back to sinus rhythm was also recorded and showed no evidence of vasodilatation.

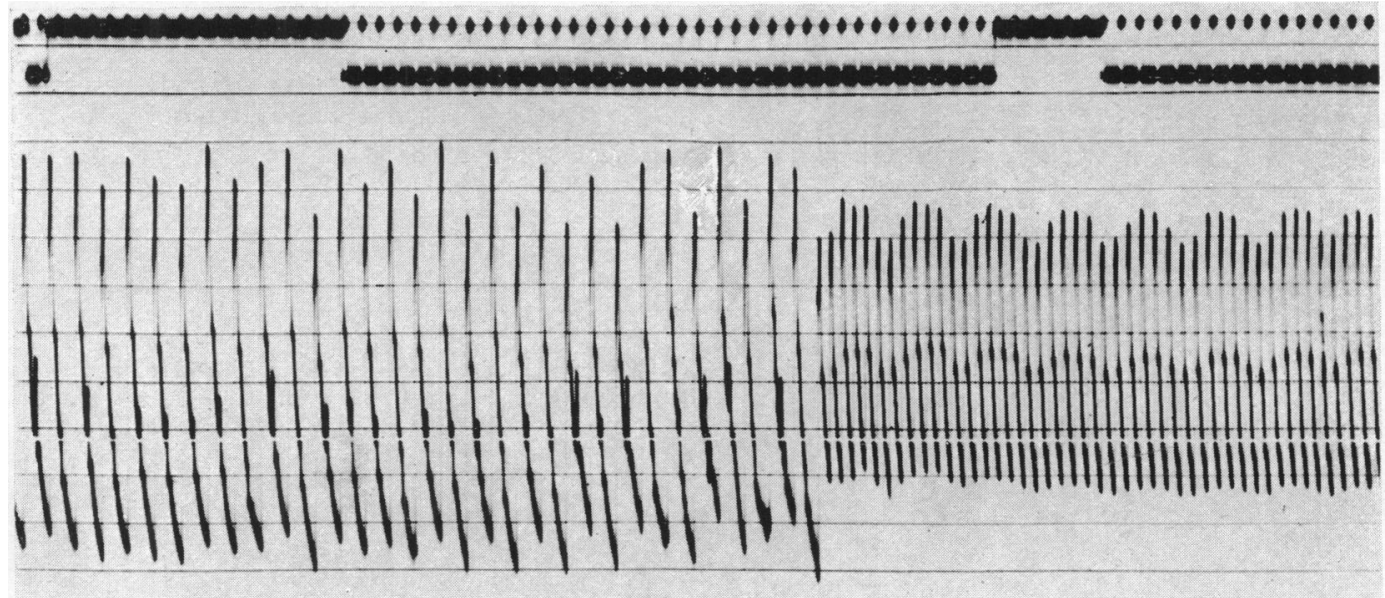

FIG. 3.-Arterial pressure curve. Hexamethonium, $50 \mathrm{mg}$., had been given a few minutes previously. Spontaneous change to a fast ectopic rhythm did not cause changes in mean arterial pressure.

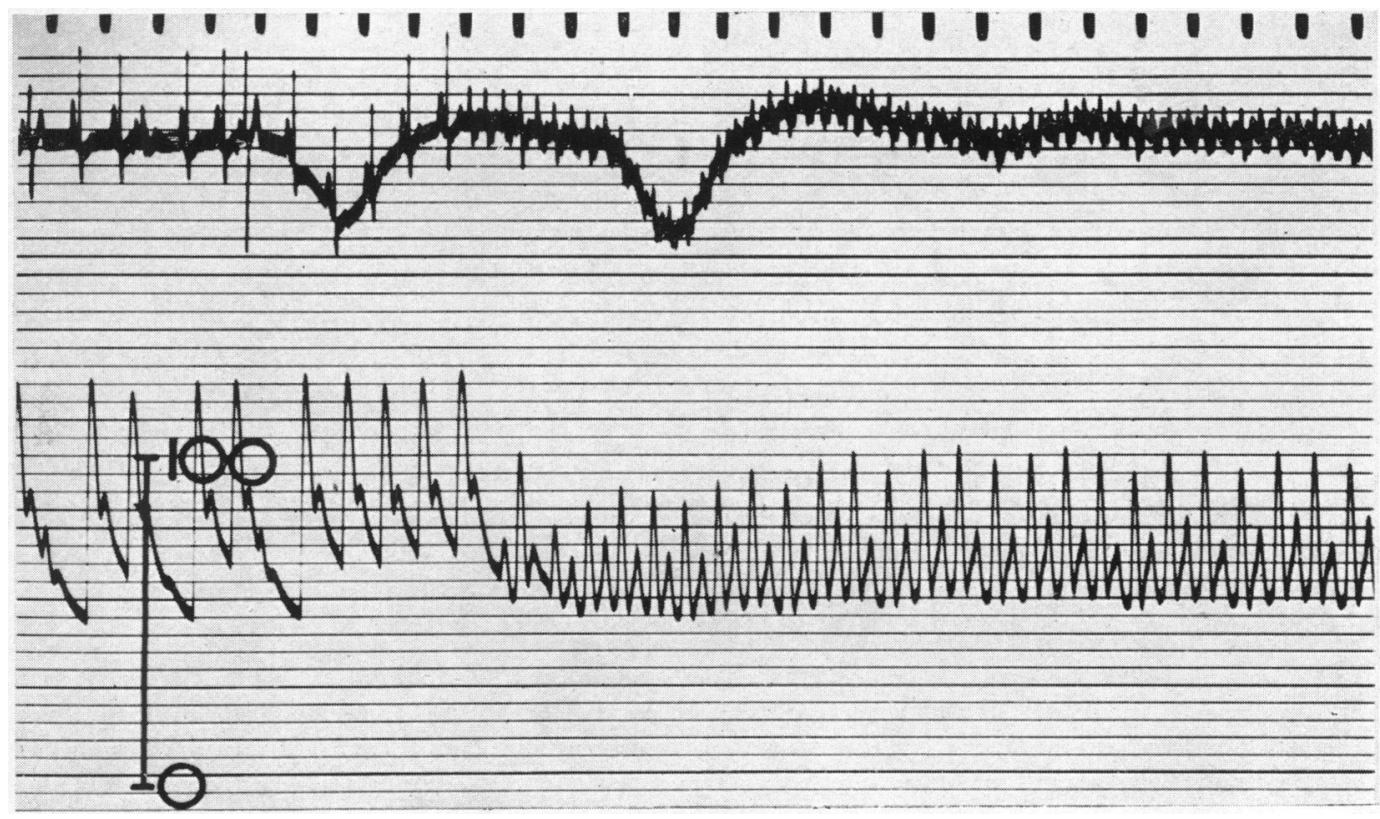

FIG. 4.-Upper trace electrocardiogram. Lower trace arterial pressure. With the change to a fast ectopic rhythm there was a fall in mean pressure and gross pulsus alternans. Rheumatic heart disease and heart failure. 


\section{Group II}

Acute Rhythm Changes in Heart Failure. Thirty-eight records were obtained in cases with heart failure. In continuous arterial records, conspicuous output changes often largely obscured baroceptor constriction or dilatation. Thus the onset of a rapid rhythm decreased minute output to such an extent that arterial mean pressure fell (Fig. 4), the changes being reversed on return to sinus rhythm (Fig. 5). Forearm flows were recorded in three cases where mean arterial pressure changes

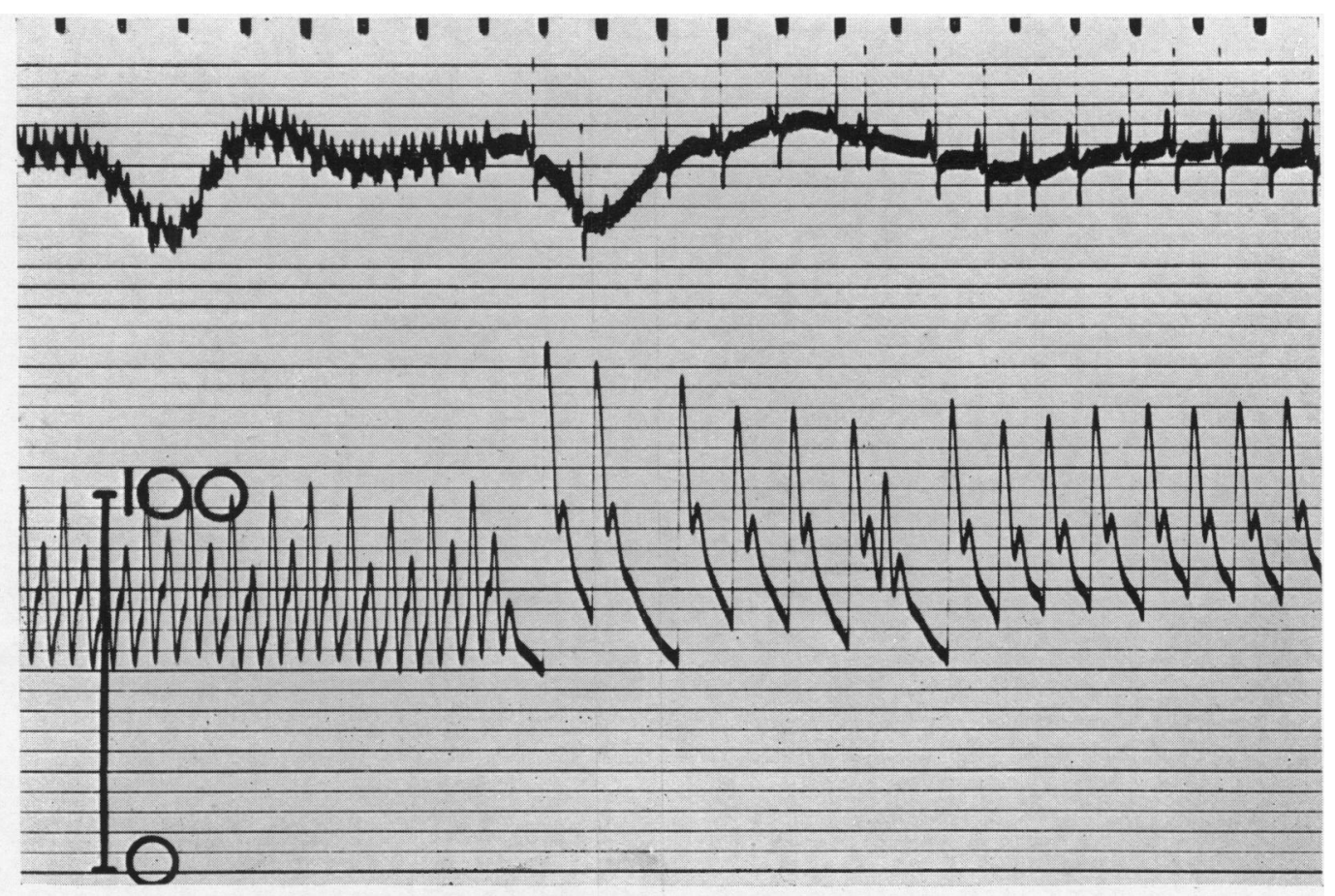

FIG. 5.-The same case as in Fig. 4, showing change back to sinus rhythm. See text.

were small. There was a great decrease in flow during the ectopic rhythm indicating vasoconstriction. In Fig. 5 it will be seen that arterial pressure is highest the moment sinus rhythm is established; thereafter it falls off as peripheral dilatation occurs in response to the increased pulse pressure.

In the most severe cases blood flow virtually ceased and arterial pressure fell rapidly below syncopal levels (Fig. 6). Death follows if the rhythm persists. In short attacks, however, the picture is that of a Stokes-Adams attack, except that the heart rate is rapid. With return to sinus rhythm there is an "overshoot" in arterial pressure similar to the "overshoot" that follows a Stokes-Adams attack (Barlow and Howarth, 1953) and indicates constriction during the attack itself.

\section{Discussion}

The results are consistent with the general theory of baroceptor responses to acute pulse pressure changes. When the first large beat occurs following the cessation of an ectopic rhythm the peripheral resistance is determined by the pulse pressure during the ectopic rhythm, and the mean pressure of this beat is unaltered. This beat, however, stimulates the baroceptors and by the time of the next beat there is demonstrable vasodilatation. Thereafter a new level of peripheral resistance is progressively established. This response to a single large beat is similar to the vasodilatation which can be demonstrated following a single cough transient (Sharpey-Schafer, 1953). 


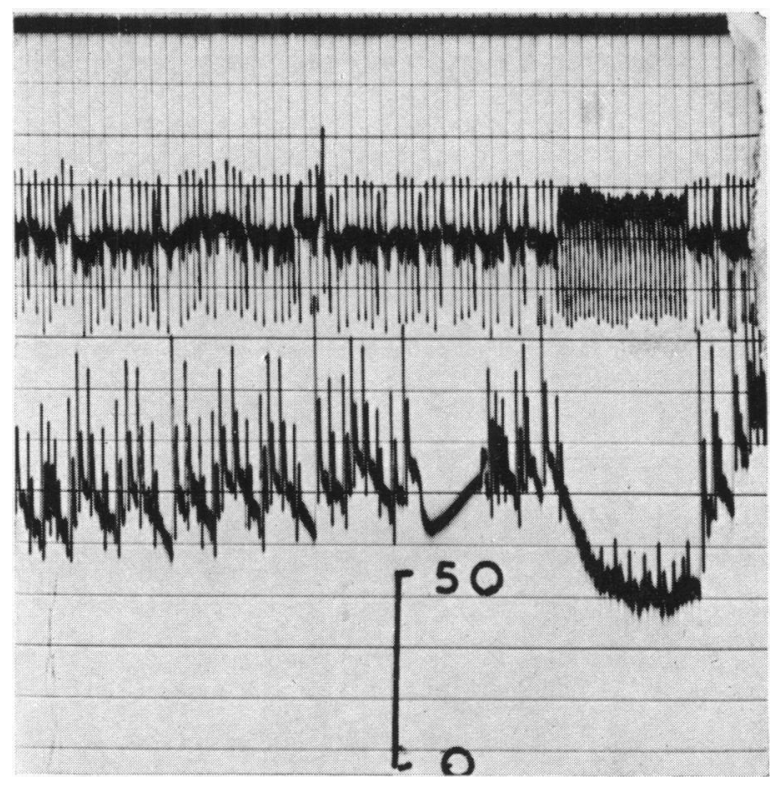

FiG. 6.-Upper trace electrocardiogram. Lower trace arterial pressure. A burst of ectopic rhythm (rate about 200 per minute) caused an acute decrease in arterial pressure and syncope. The interruption in the arterial record seen above the calibration was due to washing through the needle.

The onset of vasoconstriction with sudden decrease in pulse pressure has a rather different time relationship. In the absence of afferent impulses, the vasomotor centre is thought to exert a high level of vasoconstriction. Under normal conditions bursts of afferent impulses with each heart beat inhibit the vasomotor centre to a degree which is represented by the normal peripheral vascular resistance. Sudden increase in pulse pressure increases the inhibitory impulses and as shown above vasodilatation can be demonstrated after one such beat. Sudden decrease in pulse pressure decreases the number of afferent impulses, but the system takes time to respond. Thus there is a delay of a few seconds before constriction can be shown, and a single small beat is without demonstrable effect. This delay in the onset of constriction has been observed in other conditions: during the Valsalva manœuvre, in normal subjects, constriction does not appear for five or six seconds (Sharpey-Schafer, 1955) and on standing erect after squatting there is a similar delay (Howard et al., 1951).

The records of rhythm changes obtained under hexamethonium are those expected since the drug blocks baroceptor reflexes on the efferent pathway.

The effects of acute ectopic rhythms on the failing circulation are similar, but gross output changes largely obscure the baroceptor response in arterial records. That flutter and paroxysmal tachycardia cause intense constriction in such cases has long been known on clinical grounds. For if the abnormal rhythm persists more than a few minutes the limbs and the tip of the nose become cold and the skin cyanotic, while a conspicuous rise of venous pressure is immediate.

When stroke output falls to the lowest levels and baroceptor constriction is unable to maintain arterial pressure above $50 \mathrm{~mm}$. $\mathrm{Hg}$, syncope results.

\section{SUMMARY}

The onset and cessation of fast ectopic rhythms was studied by continuous arterial records. When the heart slowed suddenly the first large beat was followed by a fall in mean arterial pressure 
and increase in forearm blood flow indicating vasodilatation. When the heart quickened suddenly and pulse pressure decreased, mean arterial pressure rose and forearm blood flow decreased indicating vasoconstriction.

A case with a spontaneous rhythm and pulse pressure change under a sympathetic blocking agent showed no change in mean arterial pressure.

The results are compatible with the general theory of baroceptor responses to acute pulse pressure changes.

In diseased failing hearts, minute output changes with ectopic rhythms may obscure changes of peripheral resistance in continuous arterial records. Thus a fast rhythm may reduce cardiac output and arterial pressure to syncopal levels.

\section{REFERENCES}

Barlow, E., and Howarth, S. (1953). Brit. med. J., 2, 863.

Ead, H. W., Green, J. H., and Neil, E. (1952). J. Physiol. (Lond.), 118, 509.

Howard, P., Leathart, G. L., Dornhorst, A. C., and Sharpey-Schafer, E. P. (1951). Brit. med. J., $2,382$.

Sharpey-Schafer, E. P. (1953). J. Physiol. (Lond.), 122, 351.

— (1955). Brit. med. J., 1, 693.

- (1956). Brit. med. J., 1072. 